\title{
INFRASTRUCTURE READINESS FOR THE ANTICIPATED TRANSFORMATIVE CHANGES IN TRANSPORTATION
}

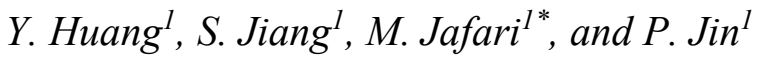 \\ ${ }^{1}$ Rutgers, The State University of New Jersey, New Brunswick, U.S. \\ * Corresponding author
}

\begin{abstract}
Connected and Autonomous Vehicles (CAVs) have gained huge expectations in improving safety, efficiency, and environmental friendliness for transportation. However, they are still at a relatively early stage of development, and little attention has been paid to the readiness of roadway infrastructure. This paper examines the issues with roadway infrastructure readiness for connected vehicles. We review two complementary system architectures for cooperative ITS (C-ITS), namely, the Connected Vehicle Reference Implementation Architecture (CVRIA) and ITS Station Architecture. Basic issues with deploying these architectures on a project basis are discussed. Gap analysis is investigated on a preliminary basis using case studies.
\end{abstract}

\section{Notation}

CAV

C-ITS

CVRIA

CEN

DSRC

ECAV

ETSI

FRAME

FCC

IoT

ISO

ITS

MIB

OBE

RSU

TMC

TIC

TCP

USDOT

UDP

V2I

$\mathrm{V} 2 \mathrm{~V}$

$\mathrm{V} 2 \mathrm{~N}$

V2P

WAVE

$5 \mathrm{G}$

5GAA

Appendix B further explains the technologies mentioned above.

\section{Introduction}

CAVs have enjoyed high expectation in improving road safety and mobility (e.g., collision avoidance, emergency response, and integrated network flow optimization). However, very few studies have focused on the readiness of the road infrastructure to support CAVs (Johnson 2017). Furthermore, little attention has been paid to how the functionality of the road infrastructure and its maintenance requirements impact on the performance of CAVs. At the same time, the idea of "clean and smart city" is increasingly embraced by cities and states in the united states, and transportation electrification is at the centre stage of these initiatives (NREL 2014). These developments are expected to bring transformative changes to transportation and energy sectors. It is widely believed that vehicle automation and ride share will be the future of the transportation, and hence, ECAV is considered the green, clean and efficient solution to that goal (Auld, Karbowski and Sokolov 2016). The success of ECAV mass deployment, however, will hinge upon roadways with clear markings, connected signage, smart traffic controls, easily accessible charging stations, and many other infrastructure related upgrades and changes (Johnson 2017). The focus of this article will be on infrastructure readiness issues and challenges facing cities and regions with mass penetration of connected vehicles. In particular, we will concentrate on system architecture and transition from legacy ITS to one that will support the anticipated transformation. We will identify the gaps and present a preliminary model to implement the C-ITS architectures.

\section{Literature Review}

Connected vehicles refer to "those that are fitted with communications devices that provide information to either the driver or the vehicle, allowing them to collaborate with other road users and parts of the road infrastructure". (Johnson 2017) Full connectedness and resilient IoT infrastructure will play 
foundational roles on ECAV mass adoption. Keeping good state of repair will be essential to traffic safety and seamless operation of the network (EuroRAP 2014). Also, social acceptance of these transformative changes will be contingent on clear policies and traffic regulations (MDOT 2017). Enabling wireless connectivity among vehicles, infrastructure, and mobile devices will boost those changes to safety, mobility, and the environmental impacts in transportation systems (Lu, et al. 2014). Commonly, the communications of connected vehicles, which is represented as $\mathrm{V} 2 \mathrm{X}$, can be generally classified into four types: (1) V2V, (2) V2I (or vice versa, I2V), (3) V2N, and (4) V2P. (5GAA 2016).

It is widely accepted that the advances of V2X communications will reshape the future of road transportation and vehicles will no longer be information-isolated (NHTSA 2014). DSRC is one of the most commonly used wireless technologies for both V2V and V2I. The U.S. FCC has applied $5.9 \mathrm{GHz}$ DSRC for road tolling (USDOT 2014). Also, DSRC beacons have been installed at several junctions to provide information including traffic conditions, traffic light signals and the positions and locations of traffic incidents or roadworks in Singapore (Land Transport Authority of Singapore 2016). However, short range communication technologies (e.g. DSRC, ZigBee and Bluetooth) are unable to handle high dynamics of network topology ( $\mathrm{Lu}$, et al. 2014). 5G is a new technology which aims at handling high data rate, reducing latency, saving energy, increasing system capacity, and solving massive device communications. It provides a new solution to increase the performance of connectivity. $5 \mathrm{G}$ will be the ultimate platform to enable C-ITS and the provision of V2X (5GAA 2016).

Better connectivity can also help increase drivers' performance in terms of reaction time and manoeuvre precision, and thus reduce accidents due to human-errors (Arbabzadeh, et al. 2019). According to USDOT, the majority of vehicles on the roadways is expected to be connected by 2040 , and up to $80 \%$ of traffic signal locations will be V2I-enabled (USDOT 2014). Accurate, real-time, localized traveller information will also be available on $90 \%$ or more of roadways at that time. Mixed vehicular technology for many years to come will certainly bring many challenges and barriers to upgrading roadway infrastructure to cope with anticipated transformations.

How ready is the current road infrastructure for connected vehicles, and what are the gaps? Here, we will address some basic aspects of these questions. We will start with different ITS architectures developed in the U.S and Europe.

\section{System Architectures}

FRAME and the U.S. National ITS Architecture are two legacy architectures that were developed to guide the development of regional ITS. (Yokota and Weiland 2004) Appendix Figure A1 illustrates their high-level physical architecture, where Travelers, vehicles, field, and centers are connected by fixed ways of communications. The information exchange is insufficient to support CAVs because vehicles cannot directly share information with roadway infrastructures, resulting in possible waste of useful data that support traffic control and decision-making. The regular cellular network mentioned in the architecture is also unable to support the large volume of traffic data nowadays (NHTSA 2014).

Currently, there are two well-developed C-ITS system architectures that are specially designed for connected vehicles. The first one is ITS Station Architecture, which is an internationally driven architecture for C-ITS. The second one is called CVRIA. As opposed to ITS Station Architecture, CVRIA is a U.S. effort. (USDOT 2014) Figure 1 shows the above four transportation architectures that are used in the U.S. and Europe. The U.S. National ITS Architecture and the FRAME are two legacy transportation architectures which are set up to guide the development of traditional ITS. While the CVRIA and the ITS Station Architecture are new for C-ITS. They set up standards for road users, roadway facilities and traffic managers to share information and better coordinate their actions by communicating with each other.

Figure 1 Difference between transportation architectures

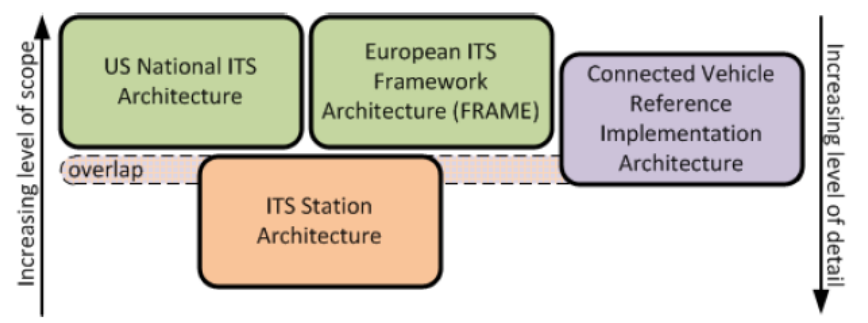

There are several differences between ITS Station Architecture and CVRIA. The former provides a set of communication standards and series of requirements for different situations and actions, while the latter focuses more on physical objects, such as RSUs, CAVs, and the TMC. Therefore, the CVRIA is less detailed in functionality compared to ITS Station Architecture, but it has a broader scope. The basic components of ITS Station Architecture are called ITS-Station Units, which are objects that contributes to C-ITS. Unlike ITS Station Architecture, the CVRIA uses physical objects to form applications, which are combinations of systems and software that provide transportation benefit. The CVRIA also considers supporting services and facilitating applications. Besides these differences, there is a similarity between the two architectures. The CVRIA defines the protocols necessary to realize a given application, which can be captured in the ITS Station Architecture. However, some standards referenced in the CVRIA cannot be found in the ITS Station Architecture, such as the message set standards (USDOT 2014).

ITS Station Architecture contains several layers. The top applications layer is designed to support multiple classes of ITS applications that rely on the communication services and vehicle operations. Based on the roadway inquiries to the system, the applications are initially grouped into "Road Safety", "Traffic Efficiency", and "Other Applications" (CALM 2016). The next layer of the ITS Station Architecture contains all blocks used to complete the communications. It 
provides standard information for common $\mathrm{V} 2 \mathrm{~V}$ and V2I applications. It also defines the communication security, connectivity, data exchange, and management functionality that implement transportation services. In addition, the security layer is responsible for authentication, authorisation and profile management (ETSI 2010). It applies firewall and hardware security modules to protect data collection, transmission, and storage.

CVRIA "identifies people and entities that have interests in CITS, frames the concerns those stakeholders have as a series of questions, and then addresses those questions in a series of views". These views are broken down to multiple applications, and then each application is modelled in graphical language to facilitate the stakeholder use (Iteris 2018). CVRIA also defines a common framework for implementation of connected vehicle functions and interfaces that is consistent with the National ITS architecture. It presents connected vehicle environment's functionalities, and information flows that enable applications and physical devices to implement them. The applications can be classified into four types: "Environmental", "Mobility", "Safety", and "Support". Table 1 lists some example applications in the CVRIA (USDOT 2017).

Table 1 Example applications in the CVRIA

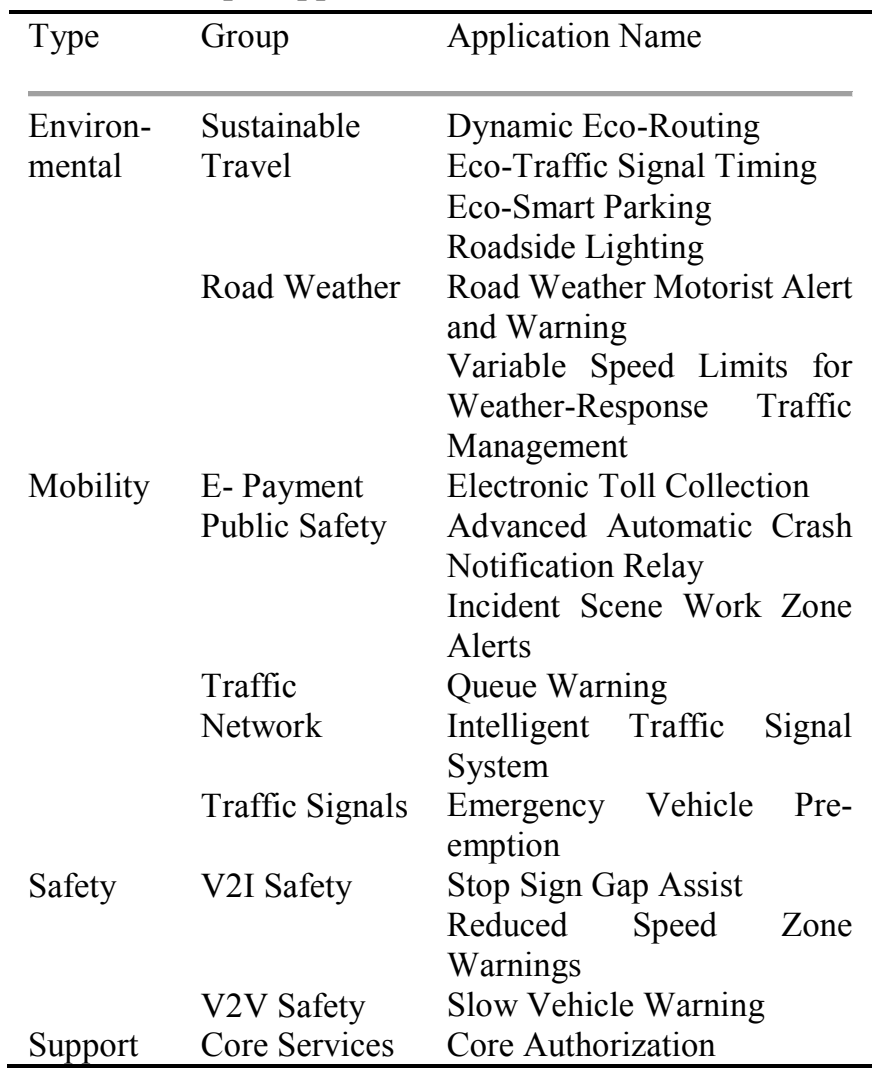

We will take the Queue Warning application as an example (see Appendix Figure A2) to demonstrate the implementation. The data going through green lines are already exist, but the red lines are currently unavailable. The diagram formulates the information flows between CAVs, RSUs, and TMC to utilize connected vehicle technologies, including V2I and V2V communications. It enables connected vehicles in the queue to automatically broadcast their status (e.g., rapid deceleration, disabled status, lane location) to nearby upstream vehicles and to infrastructure-based central entities (such as the TMC) (USDOT 2015). The infrastructure then broadcasts queue warnings to other vehicles to minimize or prevent rear-end or other secondary collisions. A vehicle's OBE can retrieve queue status form its own Databus and receive other vehicles' location and motion information from remote vehicle OBEs. It can also get driver inputs and warnings from roadside equipment. The Roadway and Roadside equipment communicate with Vehicle OBE and receive environmental sensor data. They report traffic data to the TMC and alert drivers. With data from Roadside equipment, TMC applies command and control to the ITS Roadway Equipment. It will also let other TMCs and TIC be aware of the existing congestions.

Figure 2 illustrates the ITS Station Architecture deployment. It shows how the conceptual view is mapped to a physical structure (Information Society Technologies 2009). The figure also shows how vehicles, RSUs, and the service infrastructure are connected. The ITS Station Architecture focuses more on the standardized communication requirements, but less on physical information flows. Therefore, the ITS Station Architecture is less intuitive than CVRIA, but the two architectures complement each other. For instance, the physical objects in CVRIA could be implemented as an ITSStation unit. Also, any ITS-Station Unit could be easily captured in CVRIA. The ITS Station Architecture provides a detailed logical framework for implementation, while CVRIA focuses on the realization of benefits through analysis of application concepts (USDOT 2014). These two architectures can guide local transportation agencies to build their own standards for CAVs or update their existing ITS Architectures.

Figure 2 ITS Station Architecture deployment

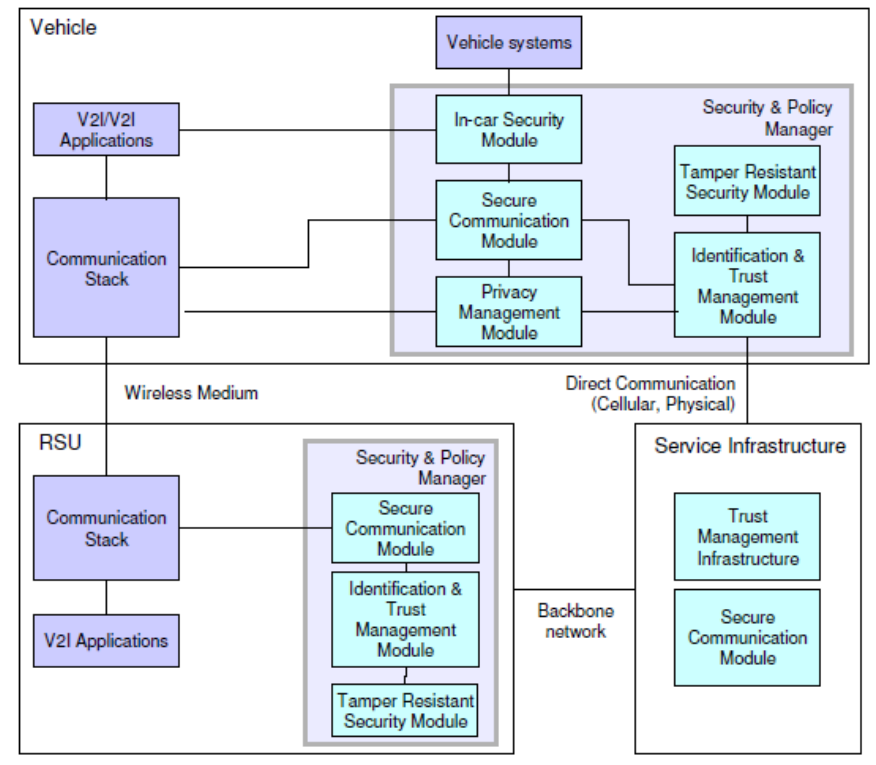




\section{Implementation of C-ITS architectures}

C-ITS architectures are specially designed to be adopted by organisations or transportation agencies to set up local C-ITS standards. The process of developing local C-ITS contains a series of systematic steps as shown in Figure 3 (Bossom and Jesty 2007). The process starts with a collection of stakeholder requirements and continues until communication specifications have been produced.

Figure 3 The process of C-ITS development

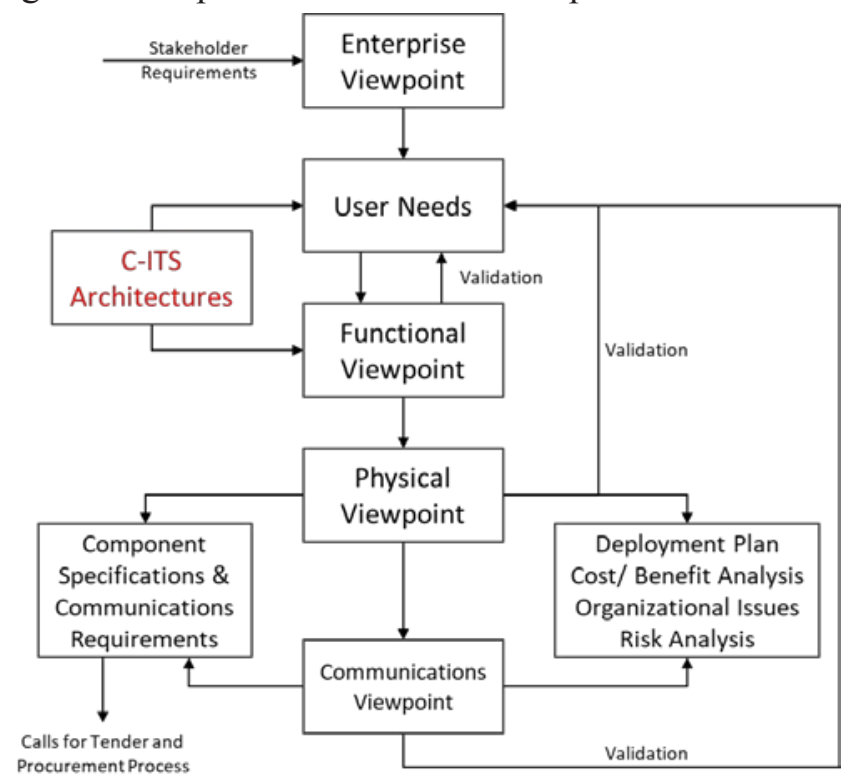

Firstly, the Enterprise Viewpoint expresses the expectations of the stakeholders on the services that the final C-ITS will provide. Then, the User Needs process is going to provide a set of clear user requirements whose properties are testable based on the stakeholder requirements. Next, the Function Viewpoint shows the functionality that is required to fulfil the users' needs. Function Viewpoints also illustrate how the functionality of the C-ITS communicate with the outside entities. After the Functional Viewpoint is established, the Physical Viewpoint will involve the allocation of functions and data to system components. Finally, the Communication Viewpoint clarifies which Function Data Flows lie within a component or pass between different sub-systems.

The C-ITS architectures take effects when defining user needs and system functionality. The ITS Station architecture provides a set of over 244 user needs to cover the C-ITS applications and services. (Bossom and Jesty 2007) Also, the CVRIA presents different service packages to address goals and objectives of regional transportation issues. (Rutgers CAIT 2018) When using these two architectures, the Functional Viewpoint is shown as a series of diagram that contains functions and the data that flows between them. Each of them includes statements explaining what they do in terms of functionality (e.g., collect and process data from connected vehicle OBEs and then produce the output data flow to Roadside Equipment). Therefore, local architecture developers only need to identify the stakeholder requirements and select parts of the Function Viewpoints that correspond to the sub-set of User Needs defined in the C-ITS architectures. New functions can also be added to the architecture when new User Needs are brought up.

\section{Challenges to current roadway infrastructure}

CVRIA and ITS Station Architecture only show the standardized data links and the information needed to support connectivity. They are independent of particular technologies or supplier products. There are discernible gaps between the legacy ITS infrastructure and the one that is under development to support the C-ITS architectures. We will further discuss some aspects of these gaps using the applications of Queue Warning and In-vehicle signage.

The Queue Warning application (Appendix Figure A2) aims to maximize the utilizing the traffic network and hence increase the mobility. The Queue Warning application performs two essential tasks: (i) queue detection and prediction, and (ii) information dissemination. In order to perform these tasks, Queue Warning solutions should be both vehicle-based and infrastructure-based (Iteris 2017). In the legacy ITS architectures, vehicles and infrastructure are isolated and TMCs collect traffic data only via roadway infrastructure instruments. TMCs lack sensors or cameras to monitor real traffic conditions, hence they are not capable of doing real time traffic controls. Also, drivers receive congestion alerts broadcasted by media or smart phones, which will help them plan alternative routes ahead of time. These gaps require infrastructure enhancements w.r.t. Vehicle Situation Data Distribution, and Traveller Situation Data Dissemination (OST-R 2014). Figure 4 demonstrates how vehicle situation data are collected from connected vehicle OBEs and sent to the Situation Data Clearinghouse and authorized interested parties.

Figure 4 Enhanced vehicle situation data distribution

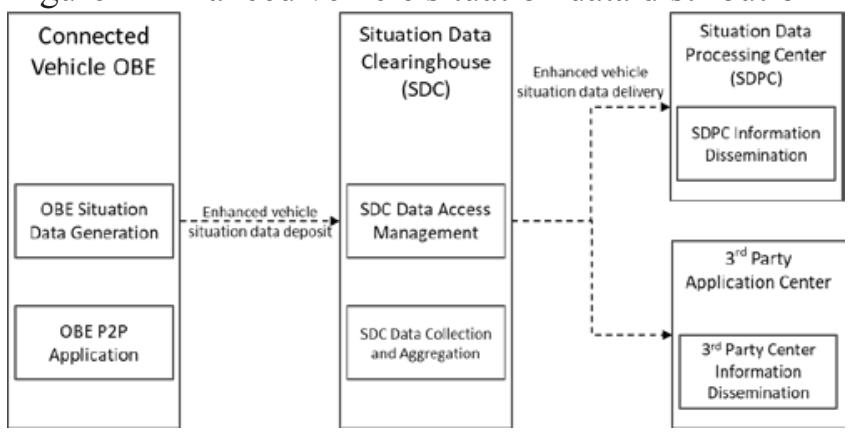

Here, the traffic data is collected from individual vehicles rather than roadway equipment. Roadway information receivers, signal repeaters, and distributed computing platform are required to realize this functionality (OST-R 2014). Meanwhile, the data volume brings challenges to the existing communication systems. Furthermore, trusted communication is an essential foundation for connectedness. It would increase the pressure on Security and Credentials Management in the system (Ramon and Zajac 2018). 
Appendix Figure A3 illustrates how traveller situation data is generated and distributed. Initially, the Situation Data Processing Center or an authorized $3^{\text {rd }}$ Party Application Center send an advisory situation data message to the Situation Data Warehouse. After the warehouse validates the message, it will then be transferred to the Roadside Equipment and Connected Vehicle Wide Area Information Distributor. Finally, the message will be directed to the Connected Vehicle OBE or Connected Vehicle Traveller Equipment to let the Driver or Traveller be aware of the traffic situation.

Data exchange is very essential to the Traveller Situation Data Dissemination system. Currently, there are two types of communication patterns to support the Data exchange (OST-R 2014). The first one is called Broadcast Communications, which applies the IEEE 1609.3 WAVE Short Message Protocol to broadcast WAVE Short Messages. The other one is called Unicast (Transactional) Communications, which apply any IP based communications path (e.g. 3G, 4G, Wi-Fi, WiMAX) for Data exchange. However, current transactions usually handle small data (approximately 10 Kbytes or less) transfers (Austroads 2018). The capability to handle larger data transfers is required. Towards this end, new communication techniques such as $5 \mathrm{G}$ will be of great needs.

The In-Vehicle Signage application is designed to improve safety in transportation. It provides roadside information directly to the drivers through in-vehicle devices. Introduced by Iteris (Iteris 2016), "the information provided would include static sign information (e.g., stop, curve warning, guide signs, service signs, and directional signs) and dynamic information (e.g., current signal states including highway intersection and highway-rail intersection status and local conditions warnings identified by local environmental sensors). This application also includes the capability for maintenance and construction and emergency vehicles to transmit sign information to vehicles in the vicinity so that in vehicle signing can be used without fixed infrastructure in work zones and around incidents."

Appendix Figure A4 demonstrates the physical diagram of the In-Vehicle Signage application. Roadside Equipment communicate with ITS Roadway Equipment, TMCs, and Parking Management Systems to gather all kinds of roadway information and deliver them to Vehicle OBEs. Also, Emergency Vehicle OBEs and Maintenance and Construction Vehicle OBEs alert the connected vehicles about the work zones ahead and incidents ahead. Therefore, Drivers can be well-informed of the incidents ahead of time and take actions accordingly.

Road markings and signages are of great significance to the performance of the application. They should be visible and clear so that they can be easily captured by the sensors and cameras equipped on connected vehicles. Notice that we are still uncertain when a significant proportion of vehicles on road will be CAVs, we might experience a mixed traffic flow on roads with both CAVs and traditional vehicles in the next 40 years (Weeratunga and Somers 2015). Therefore, drivers of the traditional cars should also be able to recognize the new designed road signs. Also, Road markings and signages should be standardized to avoid mis-representations. However, we currently do not have unified road markings (EuroRAP 2014). This makes it hard for connected vehicles to recognize some road markings that have not been standardized. Another issue to the current road markings is that they are not maintained properly and regularly. Some road markings in the rural areas become vague and hard to read (FHWA 2017). The material of road markings also needs to be tested to make sure they can survive most weather conditions in a long time. Moreover, based on the experiment done by Texas A\&M Transportation Institute, retro-reflective markings are recommended because they perform well with different brightness and weather conditions (Texas A\&M Transportation Institute 2017).

\section{Conclusions}

In this paper, we studied the infrastructure readiness for the upcoming connected vehicles from the perspective of transportation system architectures. We compared current ITS architectures and the C-ITS architectures required by the connected vehicles. Using several examples, we discussed gaps in the current roadway infrastructure. The main issue for current infrastructure is that data links do not support communication with vehicle OBEs. Also, the current communication networks lack sufficient capacity for the large volume of data created by connected vehicles. Here is a list of significant issues that require immediate attention.

(1) How can ITS Station Architecture and CVRIA be integrated to better support CAVs?

(2) How can local transportation agencies set up their own policies when applying the C-ITS architectures?

(3) Technology gaps still exist between the C-ITS architectures and their actual usage. New techniques that support safety, mobility, communication, and security need to be addressed in the development standards and kept up-to-date.

(4) Road markings and signage need to be unified in colours, sizes, and dimensions. Also, they should be maintained in good conditions.

\section{Reference}

\section{Reports}

Austroads. (2018). Connected and Automated Vehicles (CAV) Open Data Recommendations.

Bossom, R., Jesty, P. (2007). Extend FRAMEwork architecture for cooperative systems.

CALM. (2016). Communications in Intelligent Transportation System. Retrieved from http://calm.its-standards.eu/

ETSI. (2010). Intelligent Transport Systems (ITS): Communications Architecture.

EuroRAP. (2014). Roads that Cars Can Read: A Quality Standard for Road Markings and Signs on Major Rural Roads. FHWA. (2017). Rural Connected Vehicle Gap Analysis. 
Information Society Technologies. (2009). European ITS Communication Architecture.

Johnson, C. (2017). Readiness of the road network for connected and autonomous vehicles.

Land Transport Authority of Singapore. (2016). Factsheet: Facilitating the One North AV Test Bed. Retrieved from https://www.lta.gov.sg/apps/news/page.aspx?c=2\&id=9fc4a5 78-094c-4e70-84f2-d598784a3058

Lu, N., Cheng, N., Zhang, N., Shen, X., \& Mark, J. W. (2014, August). Connected Vehicles: Solutions and Challenges. IEEE Internet of Things Journal, 1, pp. 289-299.

MDOT. (2017). Automated Vehicle Industry Activities in Michigan.

NHTSA. (2014). Vehicle-to-Vehicle Communications: Readiness of V2V Technology for Application.

NREL. (2014). NREL Research and Thoughts on Connected and Automated Vehicle Energy Impacts.

OST-R. (2014). Southeast Michigan Test Bed 2014 Concept of Operations.

USDOT. (2014). CVRIA and ITS Station Primer.

USDOT. (2014). National Connected Vehicle Field Infrastructure Footprint Analysis.

VDOT. (2011). Northern Virginia ITS Architecture. Retrieved from Northern Virginia ITS Architecture: http://www.vdotitsarch.com/

Weeratunga. K. , \& Somers, A. (2015). "Connected vehicles: are we ready?"

Yokota, T., \& Weiland, R. J. (2004). ITS System Architectures for Developing Countries.

\section{Thesis}

Arbabzadeh, Nasim, Mohsen Jafari, Mohammad Jalayer, Shan Jiang, and Mohamed Kharbeche. 2019. "A Hybrid Approach for Identifying Factors Affecting Driver Reaction Time Using Naturalistic Driving Data." Transportation Research Part C: Emerging Technologies 100: 107-124.

Auld, J., Karbowski, D., \& Sokolov, V. (2016). Assessing the Regional Energy Impact of Connected Vehicle Deployment.

Lu, N., Cheng, N., Zhang, N., Shen, X., \& Mark, J. W. (2014, August). Connected Vehicles: Solutions and Challenges. IEEE Internet of Things Journal, 1, pp. 289-299.

Ramon, M. C., \& Zajac, D. A. (2018). Cybersecurity Risks of TMS and the Implications of CAV. ITS America.

Texas A\&M Transportation Institute. (2017). Road Markings for Machine Vision.

\section{Websites}

5GAA. (2016). Exploring the technology: C-V2X. Retrieved from 5GAA: http://5gaa.org/5g-technology/c-v2x/

5GAA. (2016). Vision and Mission: Building the future of connected mobility. Retrieved from 5G Automotive Association: http://5gaa.org/about-5gaa/vision-mission/
Iteris. (2016). In-Vehicle Signage. Retrieved from Connected Vehicle Reference Implementation Architecture: https://local.iteris.com/cvria/html/applications/app115.html\#t $\mathrm{ab}-3$

Iteris. (2017). Queue Warning. Retrieved from Connected Vehicle Reference Implementation Architecture: https://local.iteris.com/cvria/html/applications/app52.html\#ta b-3

Iteris. (2018). ARC-IT, The National ITS Reference Architecture. Retrieved from Views: https://local.iteris.com/arc-it/html/viewpoints/viewpoints.html

\section{Workshop}

Rutgers CAIT. (2018) Architecture Reference for Cooperative \& Intelligent Transportation. 


\section{Appendix A}

Figure A1 ITS system architecture (Yokota and Weiland 2004)

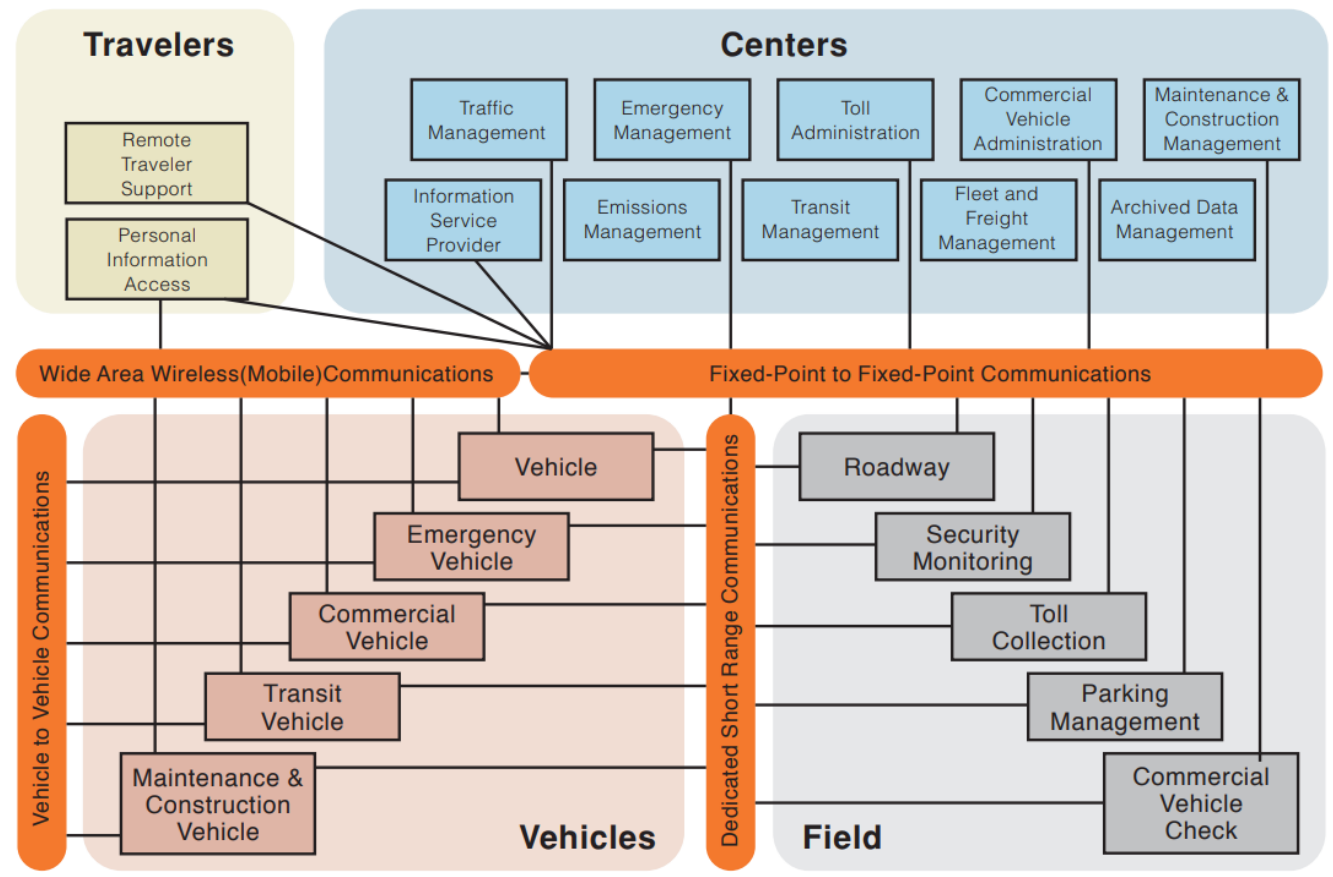

Figure A2 Queue Warning application (Iteris 2017)

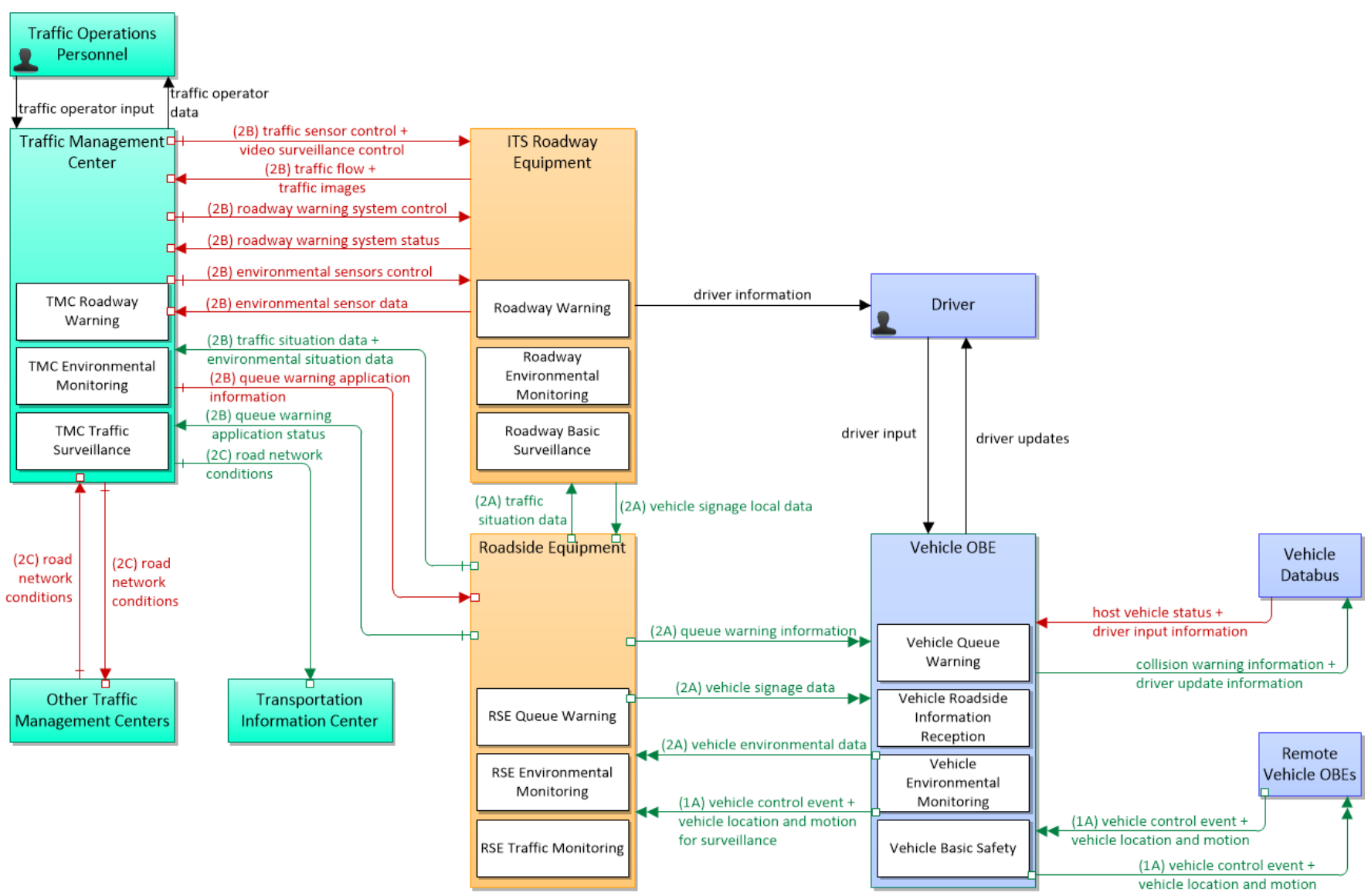


Figure A3 Traveller situation data dissemination (OST-R 2014)

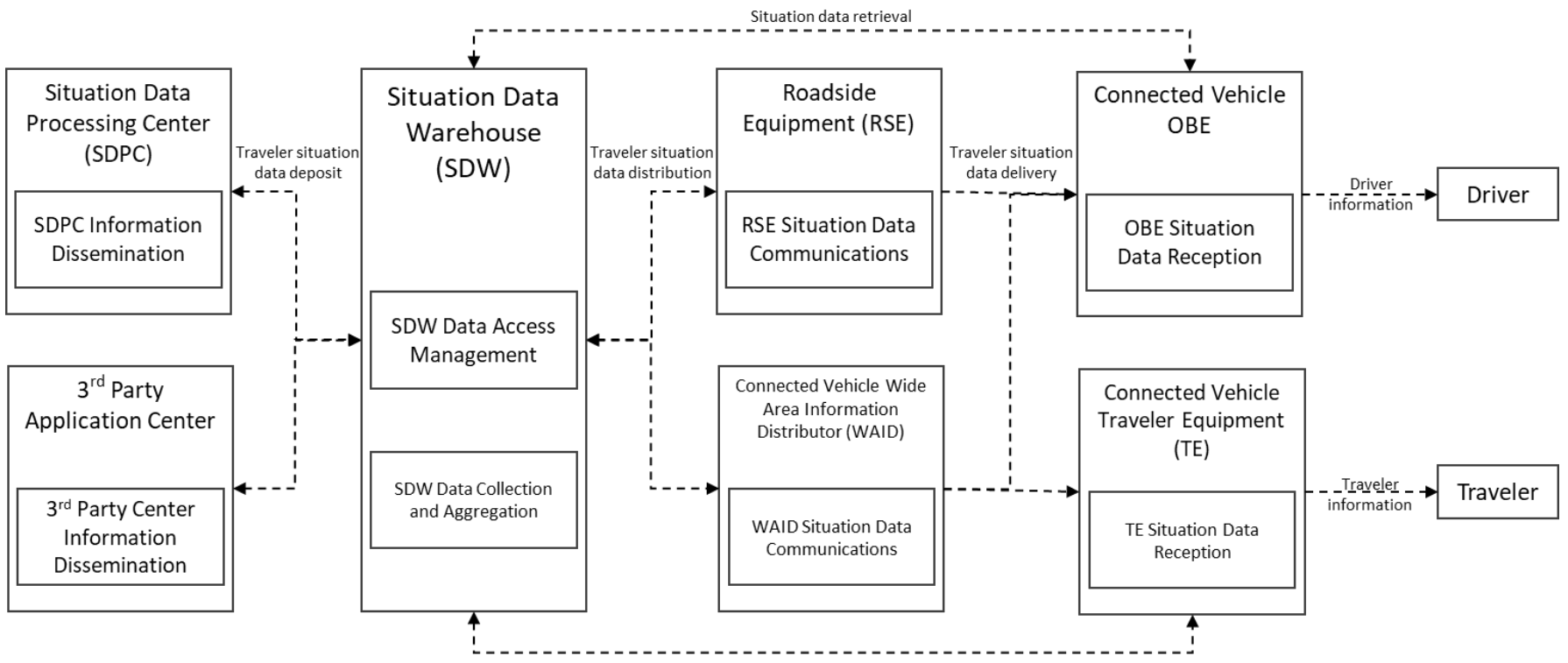

Figure A4 In-Vehicle Signage application (Iteris 2016)

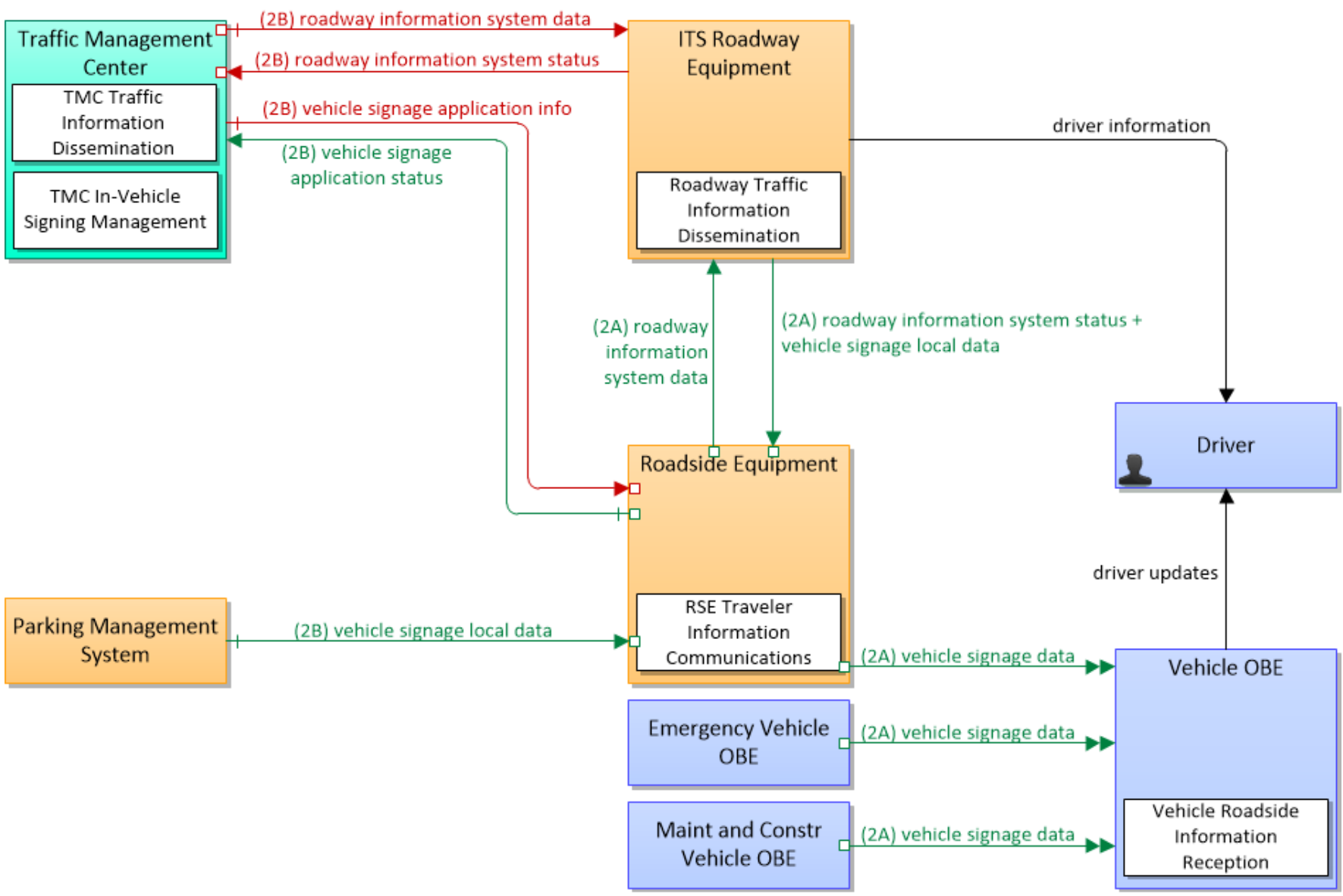




\section{Appendix B}

\begin{tabular}{|l|l|l|}
\hline Abbreviation & Full Form & Explanations \\
\hline C-ITS & $\begin{array}{l}\text { Cooperative Intelligent } \\
\text { Transport Systems }\end{array}$ & $\begin{array}{l}\text { C-ITS not only focuses on applying digital technologies to } \\
\text { provide intelligence placed at the roadside or in vehicles, } \\
\text { but also considers the communication between each vehicle, } \\
\text { roadway infrastructure, or other C-ITS systems. }\end{array}$ \\
\hline DSRC & $\begin{array}{l}\text { Dedicated Short Range } \\
\text { Communications }\end{array}$ & $\begin{array}{l}\text { DSRC is an open-source protocol for high-speed wireless } \\
\text { communications between vehicles and the infrastructure. }\end{array}$ \\
\hline IoT & Internet of Things & $\begin{array}{l}\text { IoT is a system of interrelated computing devices, mechani- } \\
\text { cal and digital machines, objects, animals or people that are } \\
\text { provided with unique identifiers and the ability to transfer } \\
\text { data over a network. }\end{array}$ \\
\hline MIB & $\begin{array}{l}\text { Management } \\
\text { Information Base }\end{array}$ & $\begin{array}{l}\text { Transmission Control } \\
\text { communication network. }\end{array}$ \\
\hline Protocol & $\begin{array}{l}\text { TCP is a standard that defines how to establish and maintain } \\
\text { a network conversation via which application programs can } \\
\text { exchange data. }\end{array}$ \\
\hline UDP & $\begin{array}{l}\text { User Datagram } \\
\text { Protocol }\end{array}$ & $\begin{array}{l}\text { UDP is an alternative communications protocol to TCP used } \\
\text { primarily for establishing low-latency and loss-tolerating } \\
\text { connections between applications on the internet. }\end{array}$ \\
\hline
\end{tabular}

